\title{
Design on a Composite Mobile System for Exploration Robot
}

\author{
Weiyan Shang, ${ }^{1,2}$ Canjun Yang, ${ }^{1}$ Yunping Liu, ${ }^{3}$ and Junming Wang ${ }^{2}$ \\ ${ }^{1}$ State Key Laboratory of Fluid Power and Mechatronic Systems, Zhejiang University, Hangzhou 310027, China \\ ${ }^{2}$ Department of Mechanical Engineering, Ningbo University of Technology, Ningbo 315000, China \\ ${ }^{3}$ College of Information and Control, Nanjing University of Information Science and Technology, \\ Collaborative Innovation Center of Atmospheric Environment and Equipment Technology, Jiangsu 210044, China
}

Correspondence should be addressed to Weiyan Shang; shangyw@umanitoba.ca

Received 6 October 2015; Accepted 6 December 2015

Academic Editor: Rafał Burdzik

Copyright (c) 2016 Weiyan Shang et al. This is an open access article distributed under the Creative Commons Attribution License, which permits unrestricted use, distribution, and reproduction in any medium, provided the original work is properly cited.

In order to accomplish exploration missions in complex environments, a new type of robot has been designed. By analyzing the characteristics of typical moving systems, a new mobile system which is named wheel-tracked moving system (WTMS) has been presented. Then by virtual prototype simulation, the new system's ability to adapt complex environments has been verified. As the curve of centroid acceleration changes in large amplitude in this simulation, ride performance of this robot has been studied. Firstly, a simplified dynamic model has been established, and then by affecting factors analysis on ride performance, an optimization model for suspension parameters has been presented. Using NSGA-II method, a set of nondominated solutions for suspension parameters has been gotten, and by weighing the importance of the objective function, an optimal solution has been selected to be applied on suspension design. As the wheel-tracked exploration robot has been designed and manufactured, the property test has been conducted. By testing on physical prototype, the robot's ability to surmount complex terrain has been verified. Design of the wheeltracked robot will provide a stable platform for field exploration tasks, and in addition, the certain configuration and suspension parameters optimization method will provide reference to other robot designs.

\section{Introduction}

An exploration robot is usually used to work in complex environments. Its abilities related to obstacle climbing, flexible steering, and passive adaptation should be improved in order to perform exploration tasks smoothly [1-3]. According to exploration conditions, the robot should be designed with the following requirements:

(1) The slope inclination angle that the robot can climb up should be no less than $35^{\circ}$; the height of barriers that it can cross over should be no lower than $160 \mathrm{~mm}$, and the width of ditches should be no less than $100 \mathrm{~mm}$.

(2) The mass of the robot should not exceed $20 \mathrm{~kg}$ and can be carried by one man. And it should be designed with following dimensions: the length $\leq 850 \mathrm{~mm}$, the width $\leq 700 \mathrm{~mm}$, and the height $\leq 400 \mathrm{~mm}$.
(3) The robot can passively adapt to different terrains and is easy to control.

\section{Structure Design}

In order to perform exploration missions in complex environments, it is very important to design the structure of the mobile system. By now, there are many types of mobile structures such as leg [4], wheel [5], track [6, 7], and some composite structures $[8,9]$. To accomplish exploration tasks efficiently, some typical mobile systems have been studied in this paper with consideration of characteristics of exploration environments.

2.1. Analysis on Typical Mobile Systems. As different structures have their own characteristics and can adapt to diverse terrains, four typical structures have been analyzed in this paper, and according to the design objects, two new 


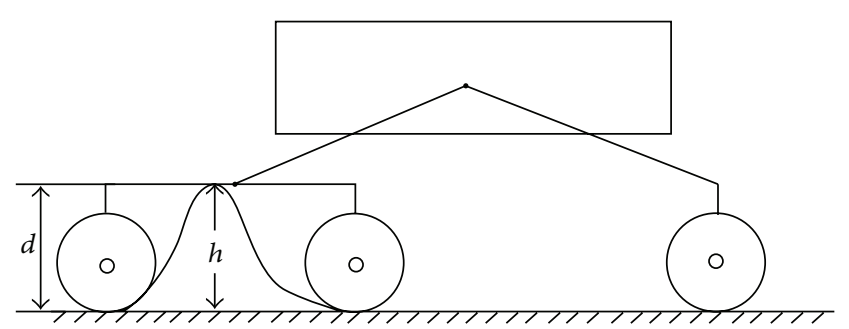

FIGURE 1: The rocker bogie mobile system.

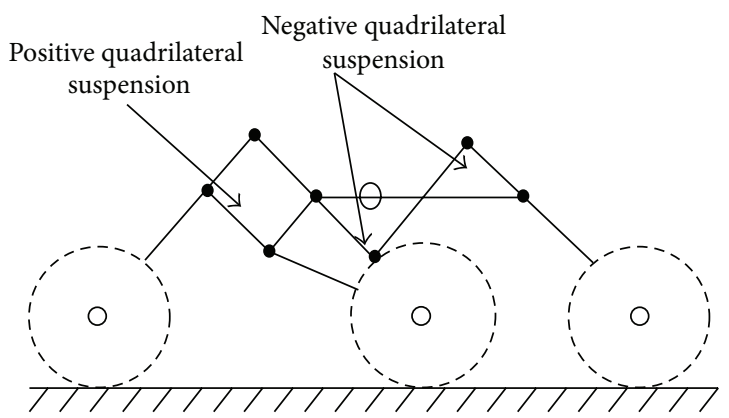

FIGURE 2: Six-wheel positive and negative quadrilateral suspension system.

structures have also been proposed. Then, we select out a suitable structure for the field exploration robot based on the six mobile systems.

2.1.1. The Wheeled-Rocker Bogie Mobile Robot. The rocker bogie mobile system has been used in space explorations [10], such as the "Sojourner" and "Spirit."

This type of mobile system can be used for field explorations for it is easy to control, has characteristics of simple structures, and can move at high speeds. There is a differential balance mechanism in wheeled-rocker bogie mobile robot. Using this mechanism, the rotation movement at left and right suspension rockers can be transferred to the bodywork. The mechanism makes the robot more stable and enables it to surmount obstacles and resist turnovers in a better way.

Because of the bogie connecting to the two front wheels, the height of the bogie should exceed the height of the obstacles, which is expressed as $d$, in order to surmount obstacles smoothly, as shown in Figure 1. For a wheeled-rocker bogie mobile robot, therefore, the ability of surmounting obstacles depends on the bogie height as well as on the wheel diameter. However, this type is not suitable for field exploration robot designs because a high bogie would also lead to a high center of gravity.

\subsubsection{The Six-Wheel Suspension System and Its Derived Forms.} There are other forms derived from the six-wheel suspension system. As shown in Figure 2, it is a positive and negative quadrilateral suspension structure [11, 12]. Figure 3 shows that it has a double crank slider suspension system. By using these suspension systems, the ability of surmounting obstacles is no longer limited to the suspension height, and the ability for

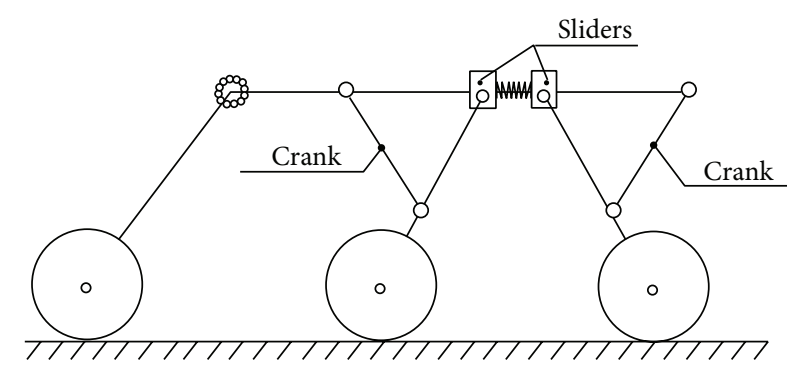

FIGURE 3: Six-wheeled and double crank slider suspension system.

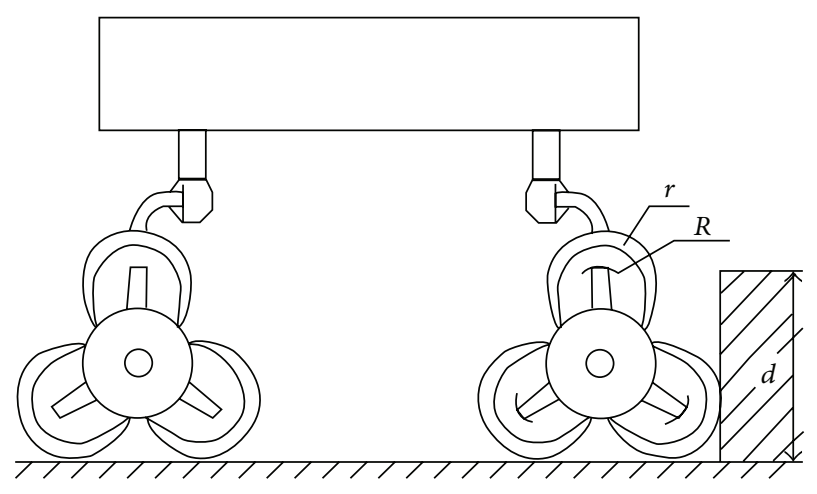

Figure 4: The planetary wheeled mobile system.

passive terrain adaptation is also improved. But the derived forms always have complex structures, and the ability of surmounting obstacles is sometimes still limited to wheel diameters, so these systems can not be widely used for field explorations.

2.1.3. The Planetary Wheeled Mobile System. Planetary wheeled mobile system [13] is shown in Figures 4 and 5. Each of the planetary wheels is driven by a motor independently. The system includes central gears, transition gears, driven gears, wheels, and connecting rods. When moving ahead, the central gear will be driven by the motor; then the movement at the central gear can be transmitted to the driven gear through the transition gear. As the wheels are fixed to the driven gear, they can rotate and bring the robot moving ahead. When there are obstacles, the triangle gear can rotate automatically because of frictions on the wheels, so the robot with this mobile system can adapt to complex terrains passively. The maximum obstacle height that can be conquered is $d=r+2 R \sin 60^{\circ}$. This type of mobile system can climb stairs and other complex obstacles, but its climbing ability on slopes should be improved.

2.1.4. The Tracked Mobile System. There are some forms derived from the tracked robot, as this type of mobile system can adapt to complex environments and has good ability of maneuverability. It has been widely used for field explorations. The MROBOT-I was designed by Nanjing University of Science and Technology [14, 15], as shown in Figure 6. 


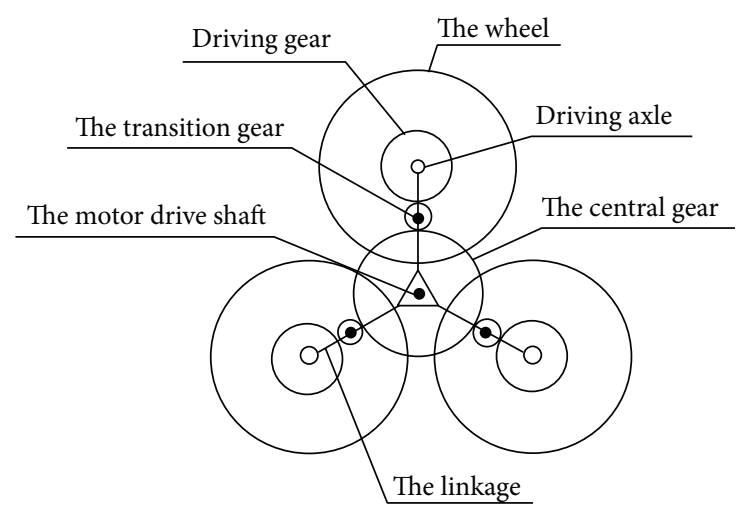

FIGURE 5: Structure of the planetary wheeled system.

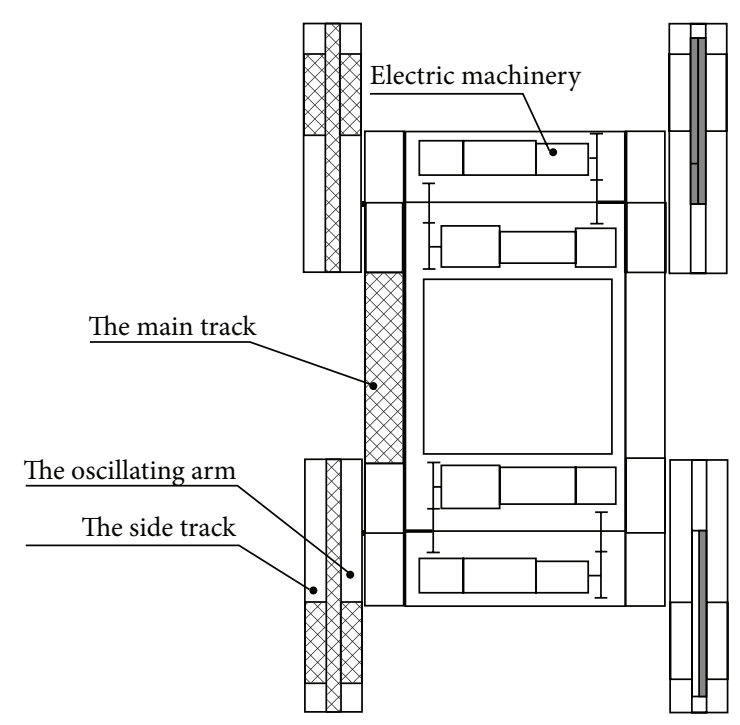

FIGURE 6: Structure of the tracked mobile system MROBOT-I.

In this system, there are oscillating arms at both sides. The robot can adapt to different terrains due to the rotation of the oscillating arms which are interactive with the tracked wheels. Some other tracked mobile systems have structures that are different from the MROBOT-I, but they all need to improve their passive adaption ability in complex terrains.

\subsubsection{The Wheel-Legged Mobile System. The wheel-legged} mobile system can adapt to complex environments by changing the posture of the legs. Its moving efficiency is improved because of the wheels installed at the end of the legs.

By mechanism synthesis [16], a six-wheel robot can be designed, shown in Figure 7.

In this exploration robot, there are two assistant wheellegs in the head. As the assistant wheel-leg is a four-bar linkage mechanism, it can rotate to the top of obstacles and help the robot to stride across complex terrains. However, this type of moving system can not adapt to uneven terrains actively, so we should search for a new moving system.

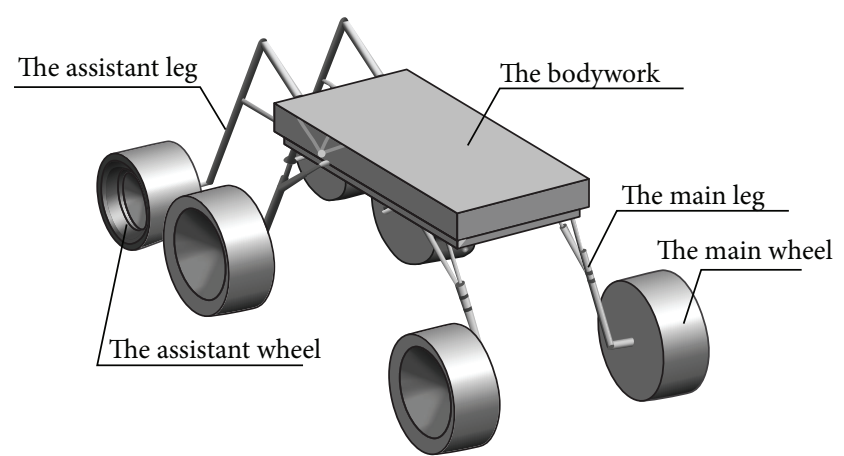

Figure 7: The model of the six-wheel-legged mobile system.

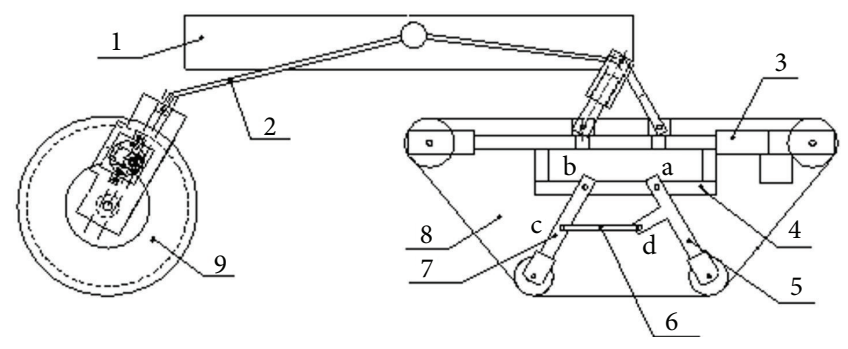
(1) Bodywork
(6) Horizontal rod
(2) Rocker suspension
(7) Side-rod
(3) Fork rod
(8) Front wheel
(4) Suspension frame
(9) Rear wheel
(5) Front rod

FIGURE 8: Schematic diagram of the wheel-tracked exploration robot.

2.1.6. The Wheel-Tracked Mobile System (WTMS). In order to combine advantages of the above mobile systems and to improve the passive adaption ability of the robot, we have designed a new type of moving system, called the wheeltracked mobile system (WTMS), as shown in Figure 8.

In this mobile system, both left and right rocker suspensions are connected to the bodywork through a differential balance mechanism, which can decrease the pitching angle of the bodywork and, accordingly, reduce impacts coming from rough terrains. Compared to the track system, this compound system can reduce the ground contact area and thus can improve the steering performance. Since the new system has the above characteristics, it is suitable for field exploration tasks.

\subsection{Simulation and Analysis to the Wheel-Tracked Exploration} Robot by Virtual Prototype. In order to validate the locomotion ability of the wheel-tracked exploration robot in rough terrains, we build a 3D simulation model of the robot by using Recurdyn and carry out the dynamic simulation in a virtual environment, as shown in Figure 9.

We can see from the simulation that the passing ability of the wheel-tracked exploration robot is better and its components work well without interference. In this simulation, the maximum height of barriers is $180 \mathrm{~mm}$, the maximum width 


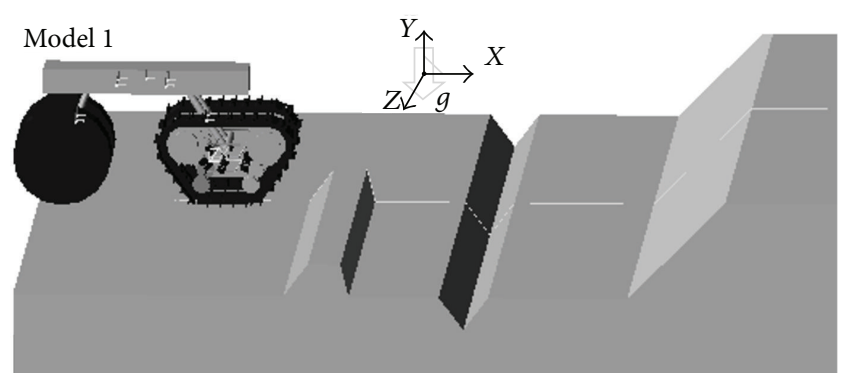

FIGURE 9: Virtual prototype of the wheel-tracked exploration robot.

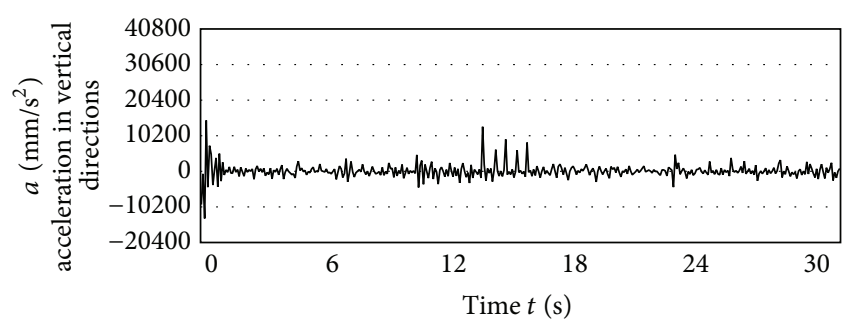

FIGURE 10: Acceleration curve of the bodywork centroid in vertical direction.

of the channel is $150 \mathrm{~mm}$, and the maximum angle of the slope is $40^{\circ}$. This simulation verifies that the new system is able to adapt to complex environments.

From the simulation we also get the acceleration curve of body center mass in vertical direction, as shown in Figure 10.

From the curve we see that the acceleration change in amplitude is large, which is mainly caused by uneven road conditions. In order to provide a good environment for automotive instruments and equipment, parameters of the wheeltracked mobile robot's suspension should be optimized to get a stable platform for field explorations.

\section{Study on the Ride Performance of the Wheel-Tracked Exploration Robot}

Ride performance is an important vehicle property. For a vehicle, good ride performance can make passenger comfortable, ensure goods not to be damaged in transportation, and prolong components' fatigue life. In the exploration robot, good ride performance is also very important for exploration tasks. Therefore, making study on the ride performance of the wheel-tracked exploration robot is very important to improve its exploration abilities.

Firstly, we should analyze the factors infecting the ride performance of the wheel-tracked exploration robot. When the robot walking on an uneven road, the road roughness and the robot's speed will be the input of this system. The input is then transferred by the vibration system which consists of rear wheel, front wheel, suspension and unsprung mass, and other elastic and damping components. Finally, the acceleration at centroid position is obtained as the output, and how to control the body output acceleration in a certain circumscription should be discussed.

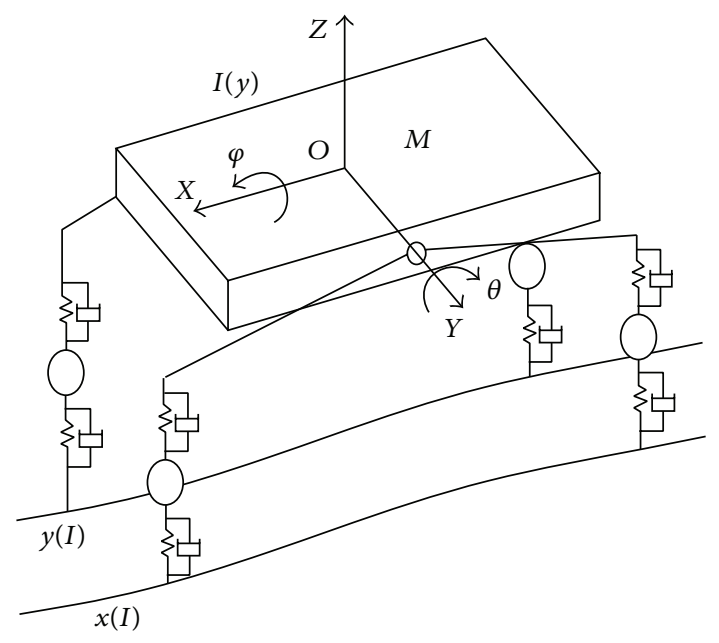

FIGURE 11: 3D dynamic simplification model of the wheel-tracked exploration robot.

3.1. System Simplification and Establishment of Dynamic Model. The structure of the wheel-tracked robot should be simplified as it is a complex vibration system. Figure 11 shows a three-dimensional simplification model. It can be seen from Figure 11 that the suspension mass consists of three components' masses, namely, the bodywork, the differential balance mechanism, and the automotive instrument. And there is also the vertical motion $(z)$, pitching motion $(\theta)$, and roll motion $(\varphi)$ in the system. And inertia moment of the suspended mass around $y$-axis is $I(y)$. The suspension mass is directly connected to the rocker suspension. The rear wheel and the front wheel are connected to the rocker suspension through the spring damping. A vehicle has seven freedoms: vertical motion, pitching motion, and roll motion, and the other four vertical degrees. The roughness function $x(I)$ approximates to $y(I)$ if the left wheel and right wheel are walking on a road with similar conditions, and there are only vertical vibration and pitch swinging that have impacts on the ride performance of the robot. In the paper, only the vertical vibration that has impacts on the ride performance has been discussed, for the pitch swinging can be solved by kinematic equations.

3.2. Simplification Model of the Rocker Suspension. The wheel-tracked exploration robot is a symmetrical system, so the vibration system can be simplified into a plane model, as shown in Figure 12. From the figure we see that the suspension mass $M$ is connected to the rocker suspension at point $B$, and the front wheel and rear wheel are connected to the rocker suspension at points $A$ and $C$, respectively. In the simplified system, components of the front wheel constitute the unsprung mass $m_{2}$, and components of the rear wheel constitute the unsprung mass $m_{3}$. The damping is too small when compared to the elasticity existing on the front wheel and rear wheel, so it is ignored during the modeling process.

In Figure 12, the elasticity and damping at coupling positions can be simplified as $K_{1}, C_{1}, K_{4}$, and $C_{4}$, respectively. Because the mass of the rocker suspension is smaller than the 


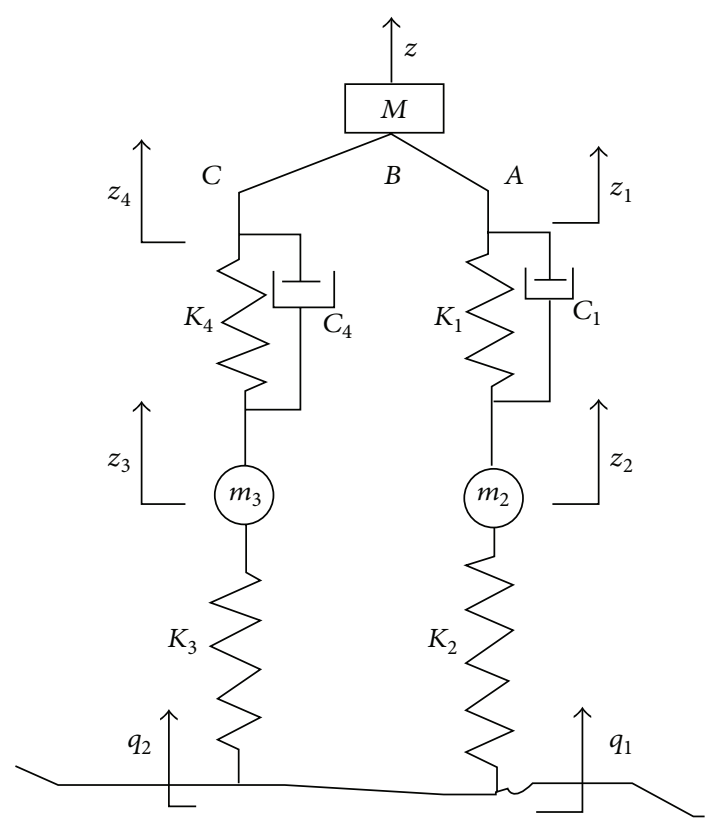

FIGURE 12: Plane model of the wheel-tracked exploration robot.

sprung mass and unsprung mass, it is regarded as a massless rod. Suppose $q_{1}(t)$ and $q_{2}(t)$ represent the pavement roughness functions of front wheel and rear wheel, respectively. Therefore, we get the following two differential equations by using Lagrange equations:

$$
\begin{aligned}
& m_{2} \ddot{z}_{2}-K_{1}\left(z_{1}-z_{2}\right)-C_{1}\left(\dot{z}_{1}-\dot{z}_{2}\right)+K_{2} z_{2}=K_{2} q_{1}, \\
& m_{3} \ddot{z}_{3}-K_{4}\left(z_{4}-z_{3}\right)-C_{4}\left(\dot{z}_{4}-\dot{z}_{3}\right)+K_{3} z_{3}=K_{3} q_{2} .
\end{aligned}
$$

To establish the vibration equation for $M$, the moving distance $z$ is expressed by $z_{1}$ and $z_{4}$. As moving distance along vertical direction of $M$ is a composite motion of points $A$ and $C$, according to the suspension structure, the linear relation among $z, z_{1}$, and $z_{4}$ can be expressed as $z=0.504 z_{1}+$ $0.419 z_{4}$

\subsection{Dynamic Model of the Front Wheel and Rear Wheel} Subsystems. As pointed in literature $[17,18]$, the vibration coming from track in a robot can decrease natural frequency of angular vibration. In the whole vibration system, the track acts as a role of damp. If the track is not considered, the vibration is more severe than it actually is. Therefore, to simplify the analysis process, the influence coming from the track is not considered, which makes the results better meet design requirements.

From Figure 8 we can see that there is a parallelogram mechanism in the front wheel. This parallelogram mechanism was jointly formed by the suspension frame, the front rod, the horizontal rod, and the side-rod. This special structure does first linear average of the hypsography and makes the exploration robot adapt to environments passively. There is a torsion spring installed between the front rod and the horizontal rod. Between the rear side-rod and the horizontal rod is also a torsion spring. The two springs in the parallelogram mechanism can help to reduce shocks

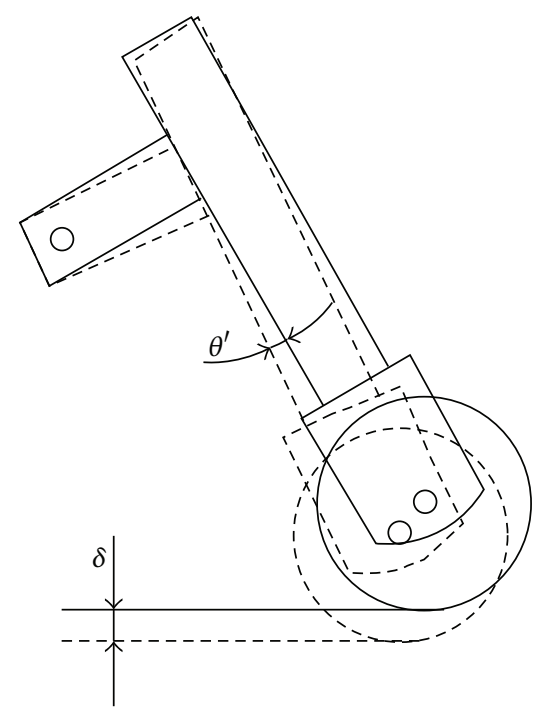

FIGURE 13: Motion position of the bearing wheel.

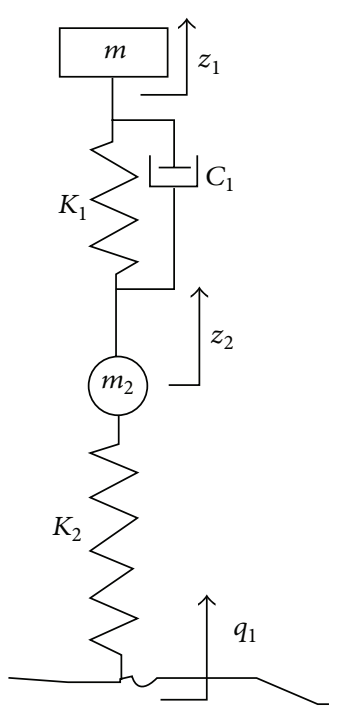

FIGURE 14: Vibration model of the front wheel.

and vibrations caused by uneven ground conditions. As the torsion spring is a nonlinear component, its effect can be equivalent to a bearing wheel.

Assume that the maximum rotate angle of the front bearing wheel is $\theta^{\prime}$, and the corresponding displacement of the bearing wheel along the vertical direction is $\delta$, as shown in Figure 13. As the maximum rotate angle for the front rod is $10^{\circ}$, the maximum value of $\delta$ is $21.03 \mathrm{~mm}$, and by using the equivalent stiffness formula

$$
K_{2}=\frac{m \cdot g}{\delta}
$$

we get the equivalent stiffness coefficient:

$$
K_{2}=7.61 \times 10^{3} \mathrm{~N} / \mathrm{m} \text {. }
$$

Figure 14 shows the simplified system of the front wheel suspension system, where there is a spring damper installed between the rocker suspension and the front wheel. 
The Kinetic equation for the front wheel system can be expressed as

$$
\begin{aligned}
& m \ddot{z}_{1}+K_{1}\left(z_{1}-z_{2}\right)+C_{1}\left(\dot{z}_{1}-\dot{z}_{2}\right)=0 \\
& m_{2} \ddot{z}_{2}-K_{1}\left(z_{1}-z_{2}\right)-C_{1}\left(\dot{z}_{1}-\dot{z}_{2}\right)+K_{2}\left(z_{2}-q_{1}\right) \\
& \quad=0
\end{aligned}
$$

where $\omega_{0}=\sqrt{K_{1} / m}, C_{1}=2 m \omega_{0} \psi_{1}$, and $\psi_{1}$ is the damping ratio of the front wheel suspension system.
Then, (5) can be simplified as

$$
\frac{z_{1}}{z_{2}}=\frac{2 \omega \omega_{0} \psi_{1} j+\omega_{0}^{2}}{-\omega^{2}+\omega_{0}^{2}+2 \omega_{0} \omega \psi_{1} j}
$$

Assume that the ratio of the spring stiffness $k_{1}$ to $k_{2}$ in front wheel suspension system is $\gamma_{1}$, and the ratio of the suspension mass $m$ to the front wheel mass $m_{2}$ is $\mu_{1}$, and there exists the equation $\lambda_{1}=\omega / \omega_{0}$.

Frequency response function can be defined as the road displacement excitation function about the displacement $z$.

Given $|H(j \omega)|_{\ddot{z}_{1}-q_{1}}=\omega^{2}\left|z_{1} / q_{1}\right|$, amplitude-frequency characteristic of $\ddot{z}_{1}$ relative to $q_{1}$ can be written as

$$
|H(j \omega)|_{\ddot{z}_{1}-q_{1}}=\sqrt{\frac{\omega^{4} \gamma_{1}^{2}\left(1+4 \psi_{1}^{2} \lambda_{1}^{2}\right)}{\left[\left(1-\lambda_{1}^{2}\right)\left(1+\gamma_{1}-\left(1 / \mu_{1}\right) \lambda_{1}^{2}\right)-1\right]^{2}+4 \psi_{1}^{2} \lambda_{1}^{2}\left[\gamma_{1}-\left(1 / \mu_{1}+1\right) \lambda_{1}^{2}\right]^{2}}} .
$$

Using the linear relationship between $z, z_{1}$, and $q_{1}$, the amplitude-frequency characteristic of $\ddot{z}$ relative to $q_{1}$ can be written as

$$
\left|\frac{\ddot{z}}{q_{1}}\right|=0.504 \sqrt{\frac{\omega^{4} \gamma_{1}^{2}\left(1+4 \psi_{1}^{2} \lambda_{1}^{2}\right)}{\left[\left(1-\lambda_{1}^{2}\right)\left(1+\gamma_{1}-\left(1 / \mu_{1}\right) \lambda_{1}^{2}\right)-1\right]^{2}+4 \psi_{1}^{2} \lambda_{1}^{2}\left[\gamma_{1}-\left(1 / \mu_{1}+1\right) \lambda_{1}^{2}\right]^{2}}} .
$$

At the same time, the suspension system of the rear wheel can be simplified as vibration system that has two degrees of freedom, as shown in Figure 15.
Using the same method, the amplitude-frequency characteristic of $\ddot{z}$ to $q_{2}$ can be written as

$$
\left|\frac{\ddot{z}}{q_{2}}\right|=0.419 \sqrt{\frac{\omega^{4} \gamma_{2}^{2}\left(1+4 \psi_{2}^{2} \lambda^{\prime 2}\right)}{\left[\left(1-\lambda_{2}^{2}\right)\left(1+\gamma_{2}-\left(1 / \mu_{2}\right) \lambda_{2}^{2}\right)-1\right]^{2}+4 \psi_{2}^{2} \lambda_{2}^{2}\left[\gamma_{2}-\left(1 / \mu_{2}+1\right) \lambda_{2}^{2}\right]^{2}}},
$$

where $\gamma_{2}=K_{3} / K_{4}, \mu_{2}=(M-m) / m_{3}, \omega_{0}^{\prime}=\sqrt{K_{4} / m}, \lambda^{\prime}=$ $2 \pi n / \omega_{0}^{\prime}, \psi_{2}=C_{4} / 2 m \omega_{0}^{\prime}$, and $\psi_{2}$ is the damping ratio of the rear wheel suspension system.

\subsection{Mean Square Values of System Dynamic Response to Pavement Random Input}

3.4.1. Calculation of Mean Square Value for the Body Acceleration. In the WTMS, there are two inputs $q_{1}(t)$ and $q_{2}(t)$. The two inputs come from the front wheel and rear wheel, respectively. So the spectral density of bodywork vibration response $S_{x}(f)$ can be regarded as a dual input system.

By calculation, the self-spectral density of robot centroid can be written as

$$
S_{\ddot{z}}(f)=\left(0.504\left|\frac{\ddot{z}_{1}}{q_{1}}\right|^{2}+0.419\left|\frac{\ddot{z}_{4}}{q_{2}}\right|^{2}\right) S_{q}(f) .
$$

Pavement power spectral density is mainly used to describe the statistical characteristics of road roughness. According to GB7031-87 "Vibration Input of VehiclesPavement Roughness Representation" [19], the spatial power spectral density for pavement can be expressed as

$$
S_{q}(f)=\frac{n_{0}^{2} v}{f^{2}} S_{q}\left(n_{0}\right),
$$

where $n_{0}$ is the reference spatial frequency and $n_{0}=0.1 \mathrm{~m}^{-1}$.

$S_{q}\left(n_{0}\right)$ is the road spectrum under reference spatial frequency $n_{0}$, called pavement roughness coefficient, and its unit is $\mathrm{m}^{2} / \mathrm{m}^{-1}$.

This expression is complex to calculate because the mean square value of body acceleration is $\sigma_{\ddot{z}}^{2}=2 \int_{0}^{\infty} S_{\ddot{z}}(f) d f$. For simplifying calculation, $N$-discrete frequency values whose band width is $\Delta f$ will be selected; then the calculation can 


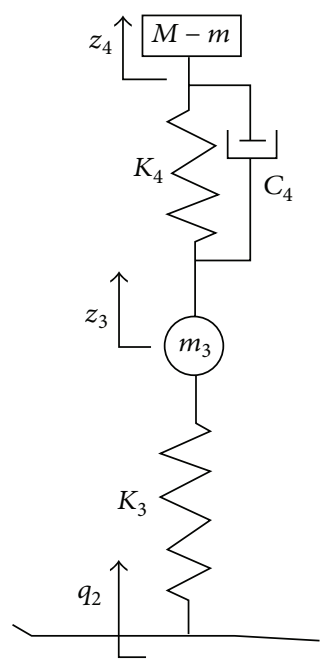

FIGURE 15: Vibration model of the rear wheel.

be conducted by numerical integration methods, as shown in the following formula:

$$
\sigma_{\ddot{z}}^{2}=2 \sum_{n=1}^{N} S_{\ddot{z}}(n \Delta f) \Delta f \quad(n=1,2,3, \ldots, N) .
$$

According to formulas (12) and (13), the mean square value of robot centroid acceleration can be expressed as

$$
\begin{aligned}
\sigma_{\ddot{z}}^{2} & =2 \sum_{n=1}^{N} S_{q}(n \Delta f) \\
& \cdot\left(0.504\left|\frac{\ddot{z}_{1}}{q_{1}}(n \Delta f)\right|^{2}+0.419\left|\frac{\ddot{z}_{4}}{q_{2}}(n \Delta f)\right|^{2}\right) \Delta f
\end{aligned}
$$

3.4.2. Calculation for Dynamic Load Mean Square Value Coming from the Front Wheel. Between the wheels and the ground exists a relative dynamic load due to the influence of uneven incentives. If the dynamic load exceeds the gravity load, the pressure acting on the ground from the wheels will be negative, which will generate influences on the adhesion and the robot can not move ahead. To improve the adhesion ability, the dynamic load acting on wheels should be controlled reasonably. Because the adhesion ability on the front wheel acts as a decisive role in the whole robot system, the dynamic load on the front track wheel needs to be investigated.
The relative load is defined as $F_{d} / F_{j}$, where $F_{d}$ represents the dynamic load and $F_{j}$ represents the static load. The mean square value of the relative load can be expressed as $\sigma_{F_{d} / F_{j}}$; if

$$
\sigma_{F_{d} / F_{j}} \geq \frac{1}{3}
$$

the direct pressure on the ground coming from the wheels is negative, which will lead to poor adhesion. And if the mean square value of the relative dynamic load is too large, the relative dynamic load acting on the axle will increase and result in wear and tear of components. So, it is important to ensure a smaller mean square value of the relative dynamic load.

From (6) and (7) we get the following formula:

$$
m \ddot{z}_{1}+m_{2} \ddot{z}_{2}=K_{2}\left(q_{1}-z_{2}\right)
$$

and there is another formula

$$
\frac{F_{d}}{m \ddot{z}_{1}}=\frac{m}{m_{2}}+\frac{z_{2}}{z_{1}} .
$$

By substituting formula (7) into formula (17), we get

$$
\frac{F_{d}}{m \ddot{z}_{1}} \frac{1}{1+\mu_{1}}=1-\frac{\omega^{2}}{\left(1+\mu_{1}\right)\left(2 \omega \omega_{0} \psi j+\omega_{0}^{2}\right)} .
$$

Given $G_{1}=\left(m+m_{2}\right) g=m\left(\mu_{1}+1\right) g$, we get

$$
\frac{F_{d}}{G_{1} q_{1}}=\frac{F_{d}}{m \ddot{z}_{1}} \frac{1}{\mu_{1}+1} \frac{1}{g} \frac{\ddot{z}_{1}}{q_{1}} .
$$

According to formulas (18) and (19), we get

$$
\begin{gathered}
\left|\frac{F_{d}}{G_{1} q_{1}}\right|=\frac{1}{g\left(\mu_{1}+1\right)}\left|\frac{F_{d}}{m \ddot{z}_{1}}\right|\left|\frac{\ddot{z}_{1}}{q_{1}}\right|=\frac{1}{g\left(\mu_{1}+1\right)} \\
\cdot \sqrt{\frac{4\left(1+\mu_{1}\right)^{2} \psi^{2} \lambda_{1}^{2}+\left(1+\mu_{1}-\lambda_{1}^{2}\right)^{2}}{4 \lambda_{1}^{2} \psi^{2}+1}\left|\frac{\ddot{z}_{1}}{q_{1}}\right| .} .
\end{gathered}
$$

The mean square value of the relative dynamic load on front track wheel is

$$
\begin{aligned}
\sigma_{F_{d} / G q_{1}}^{2}=\sum_{n=1}^{N} S_{q}(n \Delta f) \cdot\left|\frac{F_{d}}{G q_{1}}(n \Delta f)\right|^{2} \Delta f & \\
& (n=1,2,3, \ldots, N) .
\end{aligned}
$$

Finally, we get the mean square value of the relative dynamic load on the front track wheels,

$$
\sigma_{F_{d} / G q_{1}}^{2}=2 \sum_{n=1}^{N} S_{q}(n \Delta f) \cdot\left(\frac{1}{g\left(\mu_{1}+1\right)} \sqrt{\frac{4\left(1+\mu_{1}\right)^{2} \psi^{2} \lambda_{1}^{2}+\left(1+\mu_{1}-\lambda_{1}^{2}\right)^{2}}{4 \lambda_{1}^{2} \psi^{2}+1}} \mid \frac{\ddot{z}_{1}}{q_{1}}\right)^{2} \Delta f \quad(n=1,2,3, \ldots, N) .
$$

3.4.3. Analysis of the Influence of Suspension Dynamic Deflection on Ride Performance of the Wheel-Tracked Robot.
From the balance position of robot bodywork, the maximum compression stroke of suspension allowed is the limit travel 
$\left[E_{d}\right]$. Spring dynamic deflection $E_{d}$ and the limit travel $\left[E_{d}\right]$ should be properly matched; otherwise it will increase the chance of impacting limit travel when rover is running, and the ride performance of the wheel-tracked robot will get worse.

According to the structural design, spring damper of the connection position, which connects arm suspension and front track wheel suspension, creates a certain angle with vertical direction. In the simplified vibration model, the deformation in the vertical direction, which is equivalent dynamic deflection $E_{d_{1}}$, can be analyzed. The frequency response function is defined as function of the road displacement excitation relative to spring equivalent dynamic deflection $E_{d_{1}}$. The amplitude-frequency characteristic of front wheel track suspension equivalent dynamic deflection to road input can be calculated as follows:

$$
|H(j \omega)|_{E_{d} / q_{2}}=\left|\frac{E_{d_{1}}}{q_{1}}\right|=\sqrt{\frac{\gamma_{1}^{2} \lambda_{1}^{4}}{\left[\left(1-\lambda_{1}^{2}\right)\left(1+\gamma_{1}-\left(1 / \mu_{1}\right) \lambda_{1}^{2}\right)-1\right]^{2}+4 \psi_{1}^{2} \lambda_{1}^{2}\left[\gamma_{1}-\left(1 / \mu_{1}+1\right) \lambda_{1}^{2}\right]^{2}}} .
$$

When the maximum of equivalent dynamic deflection $\left|\max \left(E_{d_{1}}\right)\right|$ does not exceed the maximum limit travel $\left[E_{d_{1}}\right]$, the limiting block will not be impacted, and the ride performance will not be influenced by the impact of limiting block. However, the maximum $\left|\max \left(E_{d_{1}}\right)\right|$ of dynamic deflection is greater than the maximum limit travel $\left[E_{d_{1}}\right]$, and robot travel road conditions are usually poor, so the limiting block of suspension system will not be impacted in some cases, and the ride performance of robot will get worse. In view of the above situation, the probability $P_{1}$ of limiting block being not impacted can be calculated according to the effective values of maximum limit travel $\left[E_{d_{1}}\right]$ and $E_{d_{1}}$. The probability $P_{1}$ reflects the influence of dynamic deflection on the ride performance. As $P_{1}$ is the probability integral of standard normal distribution of ratio of $\left[E_{d_{1}}\right]$ and $\sigma_{E_{d_{1}}}$ and in the MATLAB environments, the probability in a certain range of values of random variable obeying normal probability can be calculated with normcdf function. Therefore, the probability $P_{1}$ of limiting block being not impacted can be calculated with the following formula:

$$
P_{1}=\operatorname{normcdf}\left(\frac{\left[E_{d_{1}}\right]}{\sigma_{E_{d_{1}}}}\right)
$$

However,

$$
\sigma_{E_{d_{1}}}^{2}=\sum_{n=1}^{N} S_{q}(n \Delta f) \cdot\left|\frac{E_{d_{1}}}{q_{1}}(n \Delta f)\right|^{2} \Delta f
$$

Assuming $P_{1}$ as the statistic which can be used to evaluate the influence of dynamic deflection on ride performance, the greater $P_{1}$ is, the better the index will be, and the influence of dynamic deflection on ride performance will also be better. It means the less the probability $P_{1}$ is, the worse the influence of dynamic deflection on ride performance is.

Similarly, the amplitude-frequency characteristic of front wheel track suspension equivalent dynamic deflection to road input can be calculated in the same way as follows:

$$
|H(j \omega)|_{E_{d_{2}} / q_{2}}=\left|\frac{E_{d_{2}}}{q_{2}}\right|=\sqrt{\frac{\gamma_{2}^{2} \lambda_{2}^{4}}{\left[\left(1-\lambda_{2}^{2}\right)\left(1+\gamma_{2}-\left(1 / \mu_{2}\right) \lambda_{2}^{2}\right)-1\right]^{2}+4 \psi_{2}^{2} \lambda_{2}^{2}\left[\gamma_{2}-\left(1 / \mu_{2}+1\right) \lambda_{2}^{2}\right]^{2}}} .
$$

Thus, the maximum limit travel of the rear wheel is $\left[E_{d 2}\right]=35 \mathrm{~mm}$, and the probability $P_{2}$ of the limiting block that will not be impacted can be calculated with the formula below:

$$
P_{2}=\operatorname{normcdf}\left(\frac{\left[E_{d_{2}}\right]}{\sigma_{E_{d_{2}}}}\right)
$$

\subsubsection{Establishment of the Parameter Optimization Model for} the Suspension System on the WTMS. The robot suspension flexibly connects bodywork, the front wheel and rear wheel flexibly, and it can transfer the force and torque between the bodywork and the wheels. The suspension can also reduce the impact and vibration of bearing system caused by uneven pavement, so as to ensure the ride performance of the robot and provide a stable work platform for the vehicle instrument and equipment.

The evaluation of ride performance of the robot can be achieved by optimizing spring stiffness and damping constant of damping element of suspension based on the mission execution conditions. Therefore, establishing an ideal ride performance evaluation system of robot is the basis of optimizing suspension parameters. In order to make a scientific evaluation of ride performance, the establishment of suspension parameter optimization model of the wheeltracked robot, the ascertainment of corresponding design variables, and the objective function and the constraint conditions are all necessary.

3.4.5. Establishment of Design Variables. As the quality of bodywork and front wheel track is given, and the equivalent 
stiffness coefficient between track wheel and ground is approximately constant, the stiffness coefficient and damping coefficient of the suspension will have an impact on the maximum of body acceleration and dynamic load. In order to improve the ride performance, the suspension spring stiffness coefficient and damping element of the wheels should be optimized. And the design variables of robot suspension are determined to be the equivalent stiffness coefficient of the suspension spring in front wheel track $K_{1}$, the equivalent damping coefficient $C_{1}$ of the damping element, the equivalent stiffness coefficient of the spring of rear wheel $K_{4}$, and the equivalent damping coefficient $C_{4}$ of the damping element.

3.4.6. Establishment of Objective Function. Generally, the ground condition on which the wheel-tracked robot works is complicated and harsh. However, with the designed autonomous obstacle avoidance system of robot, the real ground condition is good. Therefore, the ground condition of robot chosen is level $F, S_{q}\left(n_{0}\right)=16384$ [19]. According to the need of detecting mission, the robot usually maintains a speed of $v=0.15 \mathrm{~m} / \mathrm{s}$.
According to the above analysis, the mean square value of the body acceleration with random road input, the mean square value of the front wheel track dynamic load, and the influence of suspension dynamic deflection on ride performance of the wheel-tracked robot are, respectively, calculated and analyzed. Besides, according to the analysis of suspension mechanics, the improvement direction of the wheel dynamic load is consistent with the improvement direction of body acceleration in low frequency, while in high frequency, they improve in the opposite direction. The improvement direction of the wheel dynamic load is opposite to the improvement direction of the dynamic direction.

Therefore, those evaluation indexes of the robot ride performance are irreconcilable in the optimization process of suspension parameters. According to the analysis of factors influencing the properties, the evaluation statistics of the robot are ascertained as the effective value $\sigma_{\ddot{z}}^{2}$ and the maximum value $\max (\ddot{z})$ of body acceleration, the maximum value $\max \left(F_{d} / G_{1} q_{1}\right)$, and effective value $\sigma_{F_{d} / G_{1}}^{2}$ of dynamic load, the probability of the front and rear suspension system being not impacted $P_{1}, P_{2}$. The optimization objective function is expressed as follows:

$$
\begin{aligned}
& \min \quad \sigma_{\ddot{z}}^{2}=2 \sum_{n=1}^{N} S_{q}(n \Delta f) \cdot\left(0.504\left|\frac{\ddot{z}_{1}}{q_{1}}(n \Delta f)\right|^{2}+0.419\left|\frac{\ddot{z}_{4}}{q_{2}}(n \Delta f)\right|^{2}\right) \Delta f, \\
& \min \quad \max \left(\left|\frac{\ddot{z}}{q}\right|\right)=\left(0.504\left|\frac{\ddot{z}_{1}}{q_{1}}\right|^{2}+0.419\left|\frac{\ddot{z}_{4}}{q_{2}}\right|^{2}\right), \\
& \min \quad \sigma_{F_{d} / G}^{2}=2 \sum_{n=1}^{N} S_{q}(n \Delta f) \cdot\left(\frac{1}{g\left(\mu_{1}+1\right)} \sqrt{\frac{4\left(1+\mu_{1}\right)^{2} \psi^{2} \lambda_{1}^{2}+\left(1+\mu_{1}-\lambda_{1}^{2}\right)^{2}}{4 \lambda_{1}^{2} \psi^{2}+1}}\left|\frac{\ddot{z}_{1}}{q_{1}}\right|\right) \Delta f \\
& \min \quad \max \left(\frac{F_{d}}{G_{1} q_{1}}\right)=\frac{1}{g\left(\mu_{1}+1\right)} \sqrt{\frac{4\left(1+\mu_{1}\right)^{2} \psi^{2} \lambda_{1}^{2}+\left(1+\mu_{1}-\lambda_{1}^{2}\right)^{2}}{4 \lambda_{1}^{2} \psi^{2}+1}}\left|\frac{\ddot{z}_{1}}{q_{1}}\right| \\
& \max \quad P_{1}=\operatorname{normcdf}\left(\frac{\left[E_{d_{1}}\right]}{\sigma_{E_{d_{1}}}}\right), \\
& \max \quad P_{2}=\operatorname{normcdf}\left(\frac{\left[E_{d_{2}}\right]}{\sigma_{E_{d_{2}}}}\right) .
\end{aligned}
$$

In the optimizing process, the bandwidth $\Delta f=1 \mathrm{~Hz}$ (e.g., $n=1,2,3, \ldots, N)$ and $N=20$. The initial calculated parameters values of the robot are as follows, shown in Table 1.

3.4.7. Establishment of Constraint Conditions. In order to maintain mechanical safety, machinery, and practicality in the structural design, the design variables and system state variables must satisfy certain conditions. Those conditions are the constraints. The constraints of design variables of the wheel-tracked robot are as follows.
(1) The natural frequency of bodywork for the WTMS is

$$
f_{0}=\frac{1}{2 \pi} \sqrt{\frac{K_{1}+K_{4}}{M}} .
$$

Reducing the natural frequency $f_{0}$ of suspension system can obviously reduce the vibration acceleration of bodywork, but the dynamic deflection increases as $f_{0}$ decreases, so the reduction of $f_{0}$ is limited. The $f_{0}$ value is 1.0 1.5 for ordinary vehicles. Considering the ground condition on which the robot runs is poor, 
TABLE 1: Performance parameters of the wheel-tracked robot.

\begin{tabular}{|c|c|c|}
\hline Name of relevant parameters & Parameter values & Unit \\
\hline Suspension quality of front wheel $(m)$ & 10.5 & $\mathrm{~kg}$ \\
\hline Suspension quality of rear wheel $(M-m)$ & 13.5 & $\mathrm{~kg}$ \\
\hline Quality of front wheel track $\left(m_{2}\right)$ & 5.5 & $\mathrm{~kg}$ \\
\hline Quality of rear wheel $\left(m_{3}\right)$ & 2.5 & $\mathrm{~kg}$ \\
\hline The equivalent stiffness coefficient of the front wheel track relative to ground $\left(K_{2}\right)$ & $7.61 \times 10^{3}$ & $\mathrm{~N} / \mathrm{m}$ \\
\hline The stiffness coefficient of the rear wheel track relative to ground $\left(K_{3}\right)$ & $12.5 \times 10^{4}$ & $\mathrm{~N} / \mathrm{m}$ \\
\hline $\begin{array}{l}\text { The equivalent stiffness coefficient of the spring before optimization of the position connecting the front wheel } \\
\text { and suspension }\left(K_{1}\right)\end{array}$ & $5 \times 10^{3}$ & $\mathrm{~N} / \mathrm{m}$ \\
\hline $\begin{array}{l}\text { The equivalent damping coefficient of the damping element before optimization of the position connecting the } \\
\text { front wheel and suspension }\left(C_{1}\right)\end{array}$ & 164 & $\mathrm{Ns} / \mathrm{m}$ \\
\hline $\begin{array}{l}\text { The equivalent stiffness coefficient of the spring before optimization of the position connecting the rear wheel } \\
\text { and suspension }\left(K_{4}\right)\end{array}$ & $6 \times 10^{3}$ & $\mathrm{~N} / \mathrm{m}$ \\
\hline $\begin{array}{l}\text { The equivalent damping coefficient of the damping element before optimization of the position connecting the } \\
\text { rear wheel and suspension }\left(C_{4}\right)\end{array}$ & 200 & $\mathrm{Ns} / \mathrm{m}$ \\
\hline
\end{tabular}

the natural frequency value of suspension system of the robot with this structural design should be 2.5 3.5 to decrease the chance of impacting limiting block. Therefore, the natural frequency of the wheel-tracked robot suspension is limited as

$$
2.5 \leq f_{0}=\frac{1}{2 \pi} \sqrt{\frac{K_{1}+K_{4}}{M}} \leq 3.5 .
$$

(2) According to the provided processing conditions, the damping ratio range which may be chosen of damping element is fixed. The front robot suspension spring damping ratio $\psi$ is $0.25 \sim 0.55$. Therefore, according to the damping ratio, another constraint condition is obtained as

$$
0.25 \leq \frac{C_{1}}{2 \sqrt{m K_{1}}} \leq 0.55 .
$$

(3) Ratio $\psi_{1}$ range of the rear wheel suspension with the front wheel suspension; that is,

$$
0.25 \leq \frac{C_{4}}{2 \sqrt{(M-m) K_{4}}} \leq 0.55
$$

3.4.8. Parameter Optimization of Suspension System of the Wheel-Tracked Robot. Parameter optimization of the suspension system is a multiobjective constrained optimization problem, and the objective function is a nonlinear function. The traditional method to deal with this kind of multiobjective optimization problem is to construct an effect function and then convert the multiobjective optimization problem into a single objective optimization problem. Although the method to solve single objective optimization problem is mature, this optimization process cannot guarantee the optimality of Pareto.

Multiobjective genetic algorithm simulates the evolution process, which processes a population and generates a large number of noninferior solutions in an optimization process, so that it can find the approximate Pareto optimal solution of multiobjective optimization problems. In addition, it is a simple, general, good robustness, parallel search algorithm for global optimization processing mechanism. Pareto solutions can be defined as follows: if $x^{\prime} \in X(X$ is feasible region for the multiobjective optimization), there is not a feasible point $x \in X$, which makes the function $f_{i}(x) \geq f_{i}\left(x^{\prime}\right), i=1, \ldots, p$ ( $p$ is number of the objective function), right, and wherein at least a strict inequality is right, so $x$ is said to be an efficient solution of multiobjective optimization (also called Pareto optimal solution) [20, 21]. So we adopt a multiobjective genetic algorithm to optimize nonlinear programming problems of the robot suspension system parameter in the paper.

3.4.9. Optimizing Design of Suspension Parameter Based on NSGA-II Method. By analysis, we use NSGA-II method to optimize parameters of the suspension. As the design variables, objective function and constraint conditions have been described, the operation parameter should be set, and constraint conditions should be processed before the implementation of genetic algorithm optimization.

Set Operation Parameters. The parameters that need to be set in genetic algorithm are encoding, population size, crossover probability, mutation probability, termination of algebra, and so forth.

(1) Encoding. Adopting floating point coding, the range of the variables $K_{1}, C_{1}, K_{4}, C_{4}$ is the initial value plus $\pm 5 \%$. The initial value is

$$
X=\left(\begin{array}{llll}
K_{1} & C_{1} & K_{4} & C_{4}
\end{array}\right)=\left(\begin{array}{lllll}
5000 & 164 & 6000 & 200
\end{array}\right) .
$$

(2) Population Size $M_{c}$. If the population is too small it will be difficult to find the optimal solution, and if it is too large 
the convergence time will be prolonged. So we set $M_{c}=5 \times$ number of variables $=60$.

(3) The Crossover Probability $P_{c}$. The crossover probability $P_{c}$ is too small to search forward; while too large it is likely to damage the structure of high fitness value. The optimization using the adaptive form is $P_{c}=1.0-0.4\left(G_{i} / G_{\max }\right)$, while $G_{i}$ is the current algebra and $G_{\max }$ is the largest genetic algebra. The changing scope of the crossover probability $P_{c}$ is $(0.6 \sim 1.0)$.

(4) Mutation Probability $P_{m}$. The mutation probability $P_{m}$ is too small to produce a new gene structure, while too large genetic algorithm becomes a random simple search. So we set $P_{m}=0.0001 \sim 0.1$ and $P_{m}=0.08$ in the optimization.

(5) Termination of Algebra $G_{c}$. Generally, variable number multiplied by 200 is selected as the termination of algebra, so the system initially selected $G_{c}=400 . G_{c}$ is modified in the process of debugging the program.

Processing Constraint Conditions. The constraint conditions of design variables of optimal objective function of the wheeltracked robot are set as

(1) $2 \leq f_{0}=(1 / 2 \pi) \sqrt{\left(K_{1}+K_{4}\right) / M} \leq 3.5$,

(2) $0.25 \leq C_{1} / 2 \sqrt{m K_{1}} \leq 0.55$,

(3) $0.25 \leq C_{4} / 2 \sqrt{(M-m) K_{4}} \leq 0.65$.

Formula (1) is linear constraints which can be converted to $\mathbf{X}=\mathbf{b}$ :

$$
\left[\begin{array}{cc}
-1 & -1 \\
1 & 1
\end{array}\right]\left[\begin{array}{l}
K_{1} \\
K_{4}
\end{array}\right]=\left[\begin{array}{l}
25 \pi^{2} M \\
49 \pi^{2} M
\end{array}\right]
$$

The genetic algorithm can solve the problem with linear constraints. Formulas (2) and (3) are nonlinear constraints and can be handled with the penalty function. The idea of penalty function is imposing a penalty function when calculating the fitness of individual which has no corresponding feasible solution in solution intervals, so as to reduce the individual fitness value and make the probability of individual being genetic to the next generation population decreases. Use the following formula to adjust individual fitness:

$$
\begin{aligned}
& F^{\prime}(\mathbf{X}) \\
& = \begin{cases}F(\mathbf{X}) & \mathbf{X} \text { satisfies constraint condition } \\
F(\mathbf{X})-P(\mathbf{X}) & \mathbf{X} \text { does not satisfy constraint condition. }\end{cases}
\end{aligned}
$$

In this formula, $F(\mathbf{X})$ is the original fitness, $F^{\prime}(\mathbf{X})$ is the fitness after adjustment, and $P(\mathbf{X})$ is the penalty function. $P(\mathbf{X})=\sum g_{i}^{2}(\mathbf{X}) R_{i}(i=1,2,3,4) . R_{i}$ is penalty factor, and $R_{i}$ makes corresponding adjustment according to the degree of constraint violation, in which

$$
\begin{aligned}
& g_{1}(\mathbf{X})=0.5 \sqrt{m K_{1}}-C_{1} \leq 0 ; \\
& g_{2}(\mathbf{X})=C_{1}-1.1 \sqrt{m K_{1}} \leq 0 ; \\
& g_{3}(\mathbf{X})=0.5 \sqrt{(M-m) K_{4}}-C_{4} \leq 0 ; \\
& g_{4}(\mathbf{X})=C_{4}-1.3 \sqrt{(M-m) K_{4}} \leq 0 .
\end{aligned}
$$

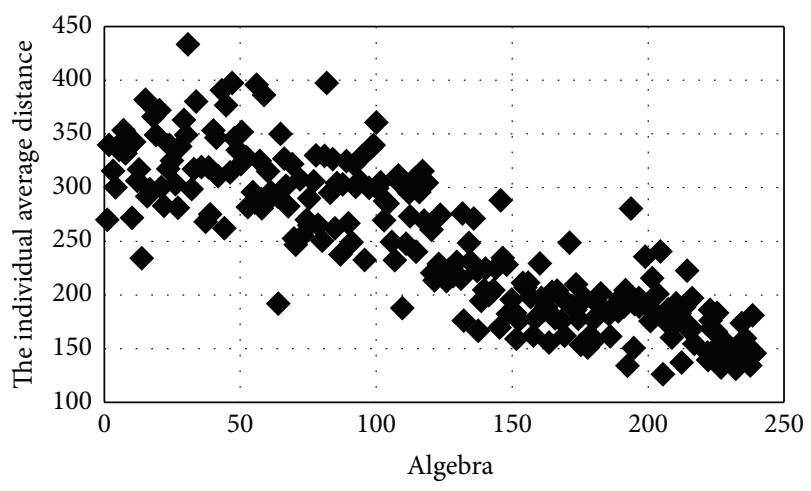

FIgURE 16: The individual average distance in the process of evolution.

3.4.10. Optimization and Analysis Results. After setting operating parameters and processing constraint condition, the design variables are optimized by NSGA-II method. The genetic algorithm meets the termination conditions and the evolution stops after 239 generations. The individual average distance is shown in Figure 16 in the process of evolution.

From Figure 16, the individual average distance significantly reduces and gradually becomes stable. The fitness of the objective function value no longer has a distinct improvement, showing that this optimization is successful.

Figure 17 is a Pareto frontier. From the figure, the 1st, 2nd, and 3rd objective function and the 4th, 5th, and 6th objective function have a competitive relationship. When one of the fitnesses of objective function improves, the fitness of objective function of another group deteriorates in different degree. Since the objective function number for the optimization is multiple and relationship is complex, the Pareto frontier of two arbitrary objective functions may not be completely smooth and uniform distribution, and it is only relatively uniform distribution. From Figure 17, the optimization process and result are satisfactory. By calculation, we get a set of nondominated solutions. According to the contrast, it can be found that the influence of different noninferior solutions on all objective fitness values is different, and a satisfactory solution can be obtained according to the importance of balance objective function. It can be seen from optimization solutions that the noninferior solutions' effect on the 5th and 6th objective function is small; therefore the influence of noninferior solution on the 5th and 6th objective function is not considered. According to the influence of noninferior solution on the 1st, $2 \mathrm{nd}$, 3rd, and 4th objective functions, the values of the objective functions 1st, 2 nd, and $3 \mathrm{rd}$ are reduced by previous set of nondominated solutions, but they greatly deteriorated the value the objective function 4th, so it is not the ideal solution that is being searched for. Balancing the influence of noninferior solution on the $3 \mathrm{rd}$ and 4th objective functions, we select the forty-seventh solution as the optimal design variables, and its corresponding design variable is (4939.173 169.7316158 .684 209.106), and the objective function value is (8985564.525 10343.313 $54861.345268 .235-0.9707-0.9523)$. This solution balances the objective functions $3 \mathrm{rd}$ and $4 \mathrm{th}$; a goal function improved performance does not deteriorate another objective function. 

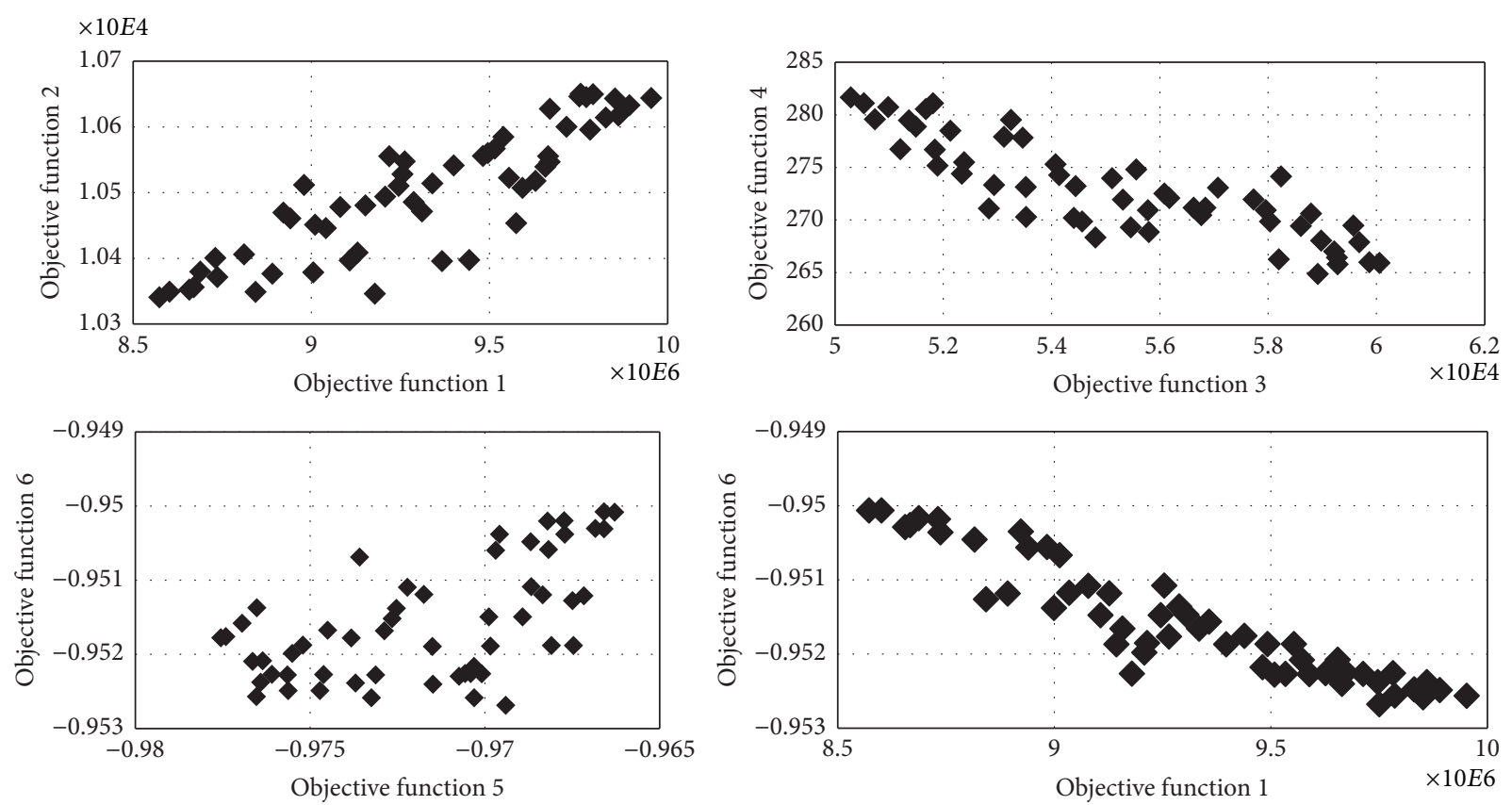

FIGURE 17: Pareto frontier.

At the same time, the solutions to the objective functions 1st and 2 nd are relatively low, and the values of the 5th and 6th objective functions are -0.9707 and -0.9523 , ensuring a high level of being not impacted rate of the suspension system.

The initial design variable $\mathbf{X}$ of the robot suspension system is (5000 1646000200$)$, and the calculated corresponding value of objective function is (9786947.726, 10593.233, $53748.859,281.4452,-0.9759,-0.9539)$. According to the contrast of the preferred solution and the initial solution, the probabilities $P_{1}$ and $P_{2}$ and the effective value $\sigma_{F_{d} / G_{1}}^{2}$ of the wheel dynamic load have been increased slightly (increased by $0.53 \%, 0.17 \%$, and $2.07 \%$, resp.). However, the effective value $\sigma_{\ddot{z}}^{2}$ and maximum value $\max (\ddot{z})$ of body acceleration and maximum value $\max \left(F_{d} / G_{1} q_{1}\right)$ of the wheel dynamic load have been decreased by $8.19 \%, 2.36 \%$, and $5.76 \%$, respectively, and the probability $P_{1}$ and $P_{2}$ can still meet the requirements as $97.07 \%$ and $95.23 \%$.

According to the above analysis, the optimization results meet the design requirement and will be referred to as the suspension design parameters of WTMS. Due to the above analysis of the stiffness and damping coefficients which are equivalent, those equivalents should be converted in the actual structure design.

\section{Physical Prototype and Tests}

According to the proposed design program and optimization parameters on the suspension system, an experimental prototype has been manufactured, shown in Figure 18, and then the performance of the wheel-tracked exploration robot has been tested.

Trench crossing test of the wheel-tracked robot was performed by gradually increasing the distance between two

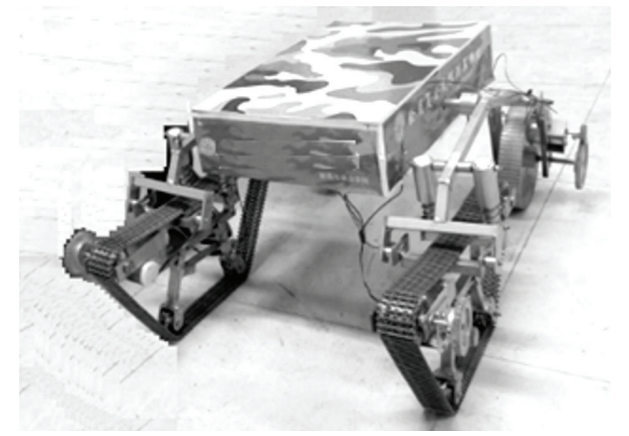

FIGURE 18: Physical prototype for wheel-tracked exploration robot.

objects. The front track wheel can smoothly cross the trench, but because of the size restrictions of the rear wheel, it becomes difficult to cross the trench gradually. After testing, the maximum width of the trench that the robot can smoothly cross is $150 \mathrm{~mm}$, and the maximum height of barriers that can be spanned is $180 \mathrm{~mm}$, which are both identical with simulation. Climbing test was carried out on indoor oblique board. The robot climbing performance is tested by changing the angle between the board and the floor. In the process of increasing board slope, the robot's climbing difficulty increases gradually. When the angle of the slope is greater than $42^{\circ}$, the climbing movement appears as the phenomenon of slipping obviously. As the maximum angle in numerical simulation is $40^{\circ}$, the robot's adhesion ability on the slope is improved compared to simulation. This is because the contact area on the track is larger than $3 \mathrm{D}$ model actually. It also shows that the bodywork is smooth in the rugged road, reflecting the robot ride performance is still good on uneven pavement condition, but further tests will be done to verify the ride performance of new suspension systems. 


\section{Conclusions}

In this paper, a new mobile type of wheel-tracked system has been designed, and by using virtual prototype, the property of this robot has been verified preliminarily. However, the ride performance of the mobile system was found to be poor by simulation, then suspension parameters optimization has been done, and, at last, the new type robot has been manufactured and the tests have been carried out. The result is as follows:

(1) The typical mobile system has been analyzed, and by combining the advantages of these systems, the wheel-tracked mobile system has been designed. Virtual simulation shows that the maximum height of barriers that the robot can cross is $180 \mathrm{~mm}$, and the maximum width of the channel that can be crossed is $150 \mathrm{~mm}$, and the wheel-tracked structure project can satisfy the basic exploration requirements.

(2) The rear wheel system and the front wheel system have been simplified; then the plan model for wheeltracked system has been established, which provides new ways for the establishment of dynamic models for complex suspension systems.

(3) Based on the system's dynamic model, ride performance of the exploration robot has been analyzed, and the optimization model has been established. By using NSGA-II method, key parameters for suspension system have been obtained. Comparing to initial suspension parameters, effective value of bodywork acceleration $\sigma_{\ddot{z}}^{2}$ has been decreased by $8.19 \%$, maximum value of bodywork acceleration $\max (\ddot{z})$ has been decreased by $2.36 \%$, and the maximum dynamic wheel load $\max \left(F_{d} / G_{1} q_{1}\right)$ has been decreased by $5.76 \%$ theoretically.

(4) According to the design structure and optimization parameters for the suspension, physical prototype has been processed and tests have been carried out accordingly. By the tests, the maximum width of the trench that the robot can smoothly cross is identical to prototype simulation, while the climbing test shows the maximum angle of the slope that can be climbed up is $42^{\circ}$, which is larger than the numerical value in prototype simulation.

\section{Conflict of Interests}

The authors declare that there is no conflict of interests regarding the publication of this paper.

\section{Acknowledgments}

This work was supported by Grant no. LQ15E050004 (study on the stability of mobile robot for seafloor exploration, supported by Natural Science Foundation of Zhejiang Province). Some of the simulation analysis was supported by Grant no. 2014A610081 (a four-legged climbing wall robot based on Flexible Biomimetic adsorption unit, supported by Natural
Science Foundation of Ningbo). The experiment work was also supported by Natural Science Foundation of Jiangsu Province (BK20130999), the Natural Science Foundation of Colleges and Universities in Jiangsu Province (13KJB460012), and the China Science Foundations (61203327, 51405243). The authors would like to express appreciation for their assistance and attention.

\section{References}

[1] S. G. Tzafestas, "Mobile robot path, motion, and task planning," in Introduction to Mobile Robot Control, chapter 11, pp. 429-478, Elsevier, 2014.

[2] H. Voos and R. Stetter, "Design and control of a mobile exploration robot," in Proceedings of the 4th IFAC Symposium on Mechatronic Systems, vol. 4, pp. 950-955, Heidelberg, Germany, September 2006.

[3] Z. R. Luo, J. Z. Shang, and Z.X. Zhang, "Innovative design of six wheeled space exploration robot using module combination," in Proceedings of the 19th International Conference on Mechatronics and Machine Vision in Practice, pp. 460-465, Auckland, New Zealand, November 2012.

[4] S. S. Roy and D. K. Pratihar, "Dynamic modeling, stability and energy consumption analysis of a realistic six-legged walking robot," Robotics and Computer-Integrated Manufacturing, vol. 29, no. 2, pp. 400-416, 2013.

[5] P. Zhang, Z. Li, Y. Hu, X. Wang, and J. Zhang, "Mobility performance analysis of an out-door mobile robot with foldable wheels," in Proceedings of the 5th International Conference on Intelligent Human-Machine Systems and Cybernetics (IHMSC '13), pp. 236-240, Hangzhou, China, August 2013.

[6] B. Zhou, J. D. Han, and X. Z. Dai, "Backstepping based global exponential stabilization of a tracked mobile robot with slipping perturbation," Journal of Bionic Engineering, vol. 8, no. 1, pp. 6976, 2011.

[7] R. Shah, S. Ozcelik, and R. Challoo, "Design of a highly maneuverable mobile robot," Procedia Computer Science, vol. 12, pp. 170-175, 2012.

[8] K. Inagaki and N. I. B. Azlizan, "Skating motion by a legwheeled robot with passive wheels," in Proceedings of the IEEE/ASME International Conference on Advanced Intelligent Mechatronics (AIM '13), pp. 524-529, Wollongong, Australia, July 2013.

[9] R. C. Luo, M. Hsiao, and T.-W. Lin, "Erect wheel-legged stair climbing robot for indoor service applications," in Proceedings of the IEEE/RSJ International Conference on Intelligent Robots and Systems (IROS '13), pp. 2731-2736, IEEE, Tokyo, Japan, November 2013.

[10] D. Kim, H. Hong, H. S. Kim, and J. Kim, "Optimal design and kinetic analysis of a stair-climbing mobile robot with rockerbogie mechanism," Mechanism and Machine Theory, vol. 50, pp. 90-108, 2012.

[11] Y. Lu, Mechanism analysis and parameter optimization of lunar rover suspension [M.S. thesis], Jilin University, Jilin, China, 2007 (Chinese).

[12] J. Z. Shang, Research on the Mobile Mechanism and System for Space Exploration Robot, Huazhong University of Science and Technology, Wuhan, 2006 (Chinese).

[13] H.-B. Gao, Q.-X. Meng, and Z.-Q. Deng, "Analysis of dynamic loads between wheels of the planetary wheel lunar rover and 
ground," Journal of Harbin Institute of Technology, no. 4, pp. 523-527, 2006 (Chinese).

[14] H. T. Feng, Y. Ou, and X. Gao, "Dynamic modeling and analysis of miniature ground moving robot based on special moving pose," Journal of Nanjing University of Science and Technology (Natural Science Edition), no. 4, pp. 486-490, 2006 (Chinese).

[15] Y. Ou, Research on Design and Dynamic Analysis of Mechanical System, Nanjing University of Technology, Nanjing, China, 2006 (Chinese).

[16] W. Y. Shang, S. M. Li, F. J. Qiu et al., "Structure design and analysis for four wheel-legged lunar rover," Journal of Wuhan University of Technology (Transportation Science \& Engineering), vol. 32, no. 5, pp. 822-825, 2008.

[17] F. G. Ding, Dynamic Analysis on Tracked Armored Vehicle Suspension System, National Defence Industry Press, Beijing, China, 2004 (Chinese).

[18] Z. C. Wu, S. Z. Chen, L. Yang et al., "Study on ride performance of off-road vehicle," Journal of Materials Engineering, vol. 28, no. 11, pp. 1393-1396, 2007 (Chinese).

[19] M. C. Lin and J. H. Zhao, GB7031-1987 Vehicle Vibration Input-Expression of Pavement Roughness, Chinese Standards Press, Beijing, China, 1987.

[20] C. M. Fonseca and P. J. Fleming, "Multiobjective optimization and multiple constraint handling with evolutionary algorithms-part I: a unified formulation," IEEE Transactions on Systems, Man, and Cybernetics, Part A: Systems, vol. 28, no. 1, pp. 26-37, 1998.

[21] J. Horn and N. Nafpliotis, "Multiobjective optimization using the niched pareto genetic algorithm," in Proceedings of the 1st IEEE Conference on Evolutionary Computation and IEEE World Congress on Computational Intelligence, pp. 1-6, Urbana, Ill, USA, 1994. 


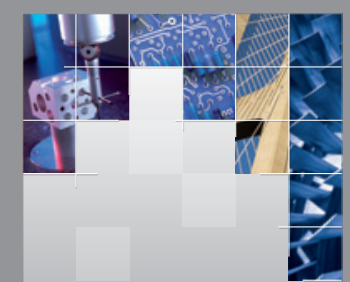

\section{Enfincering}
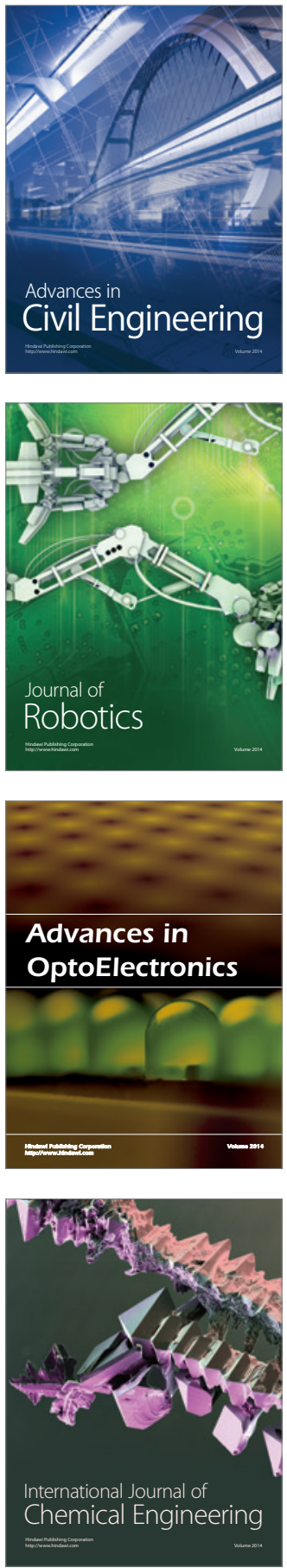

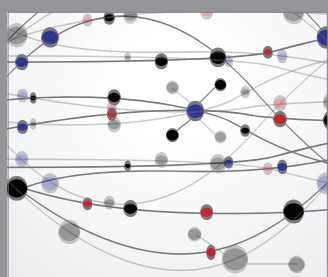

The Scientific World Journal

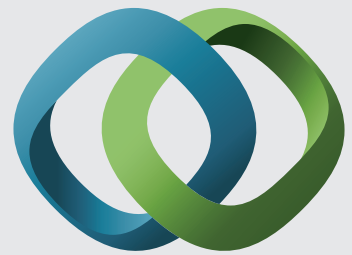

\section{Hindawi}

Submit your manuscripts at

http://www.hindawi.com
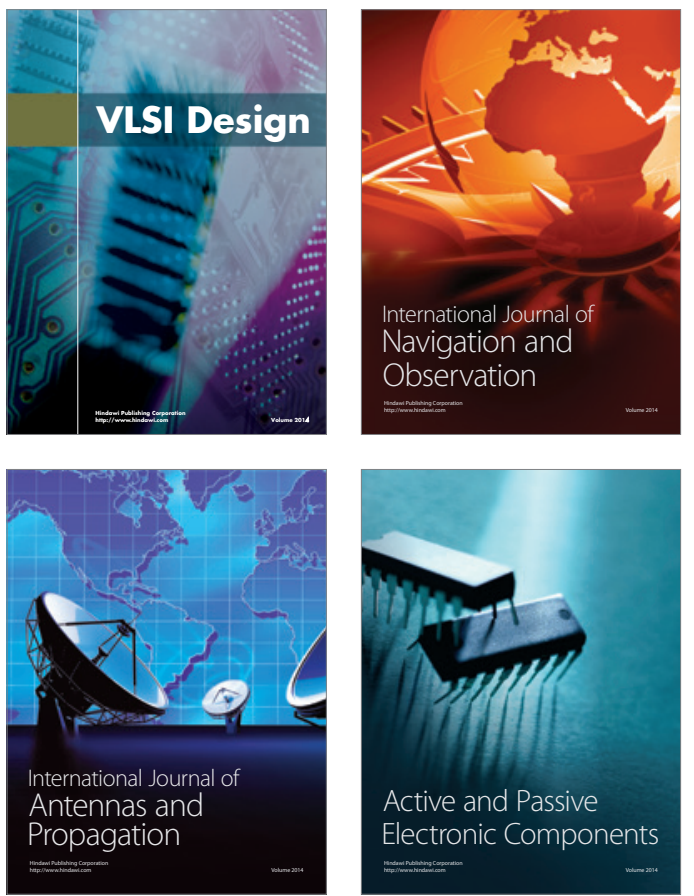
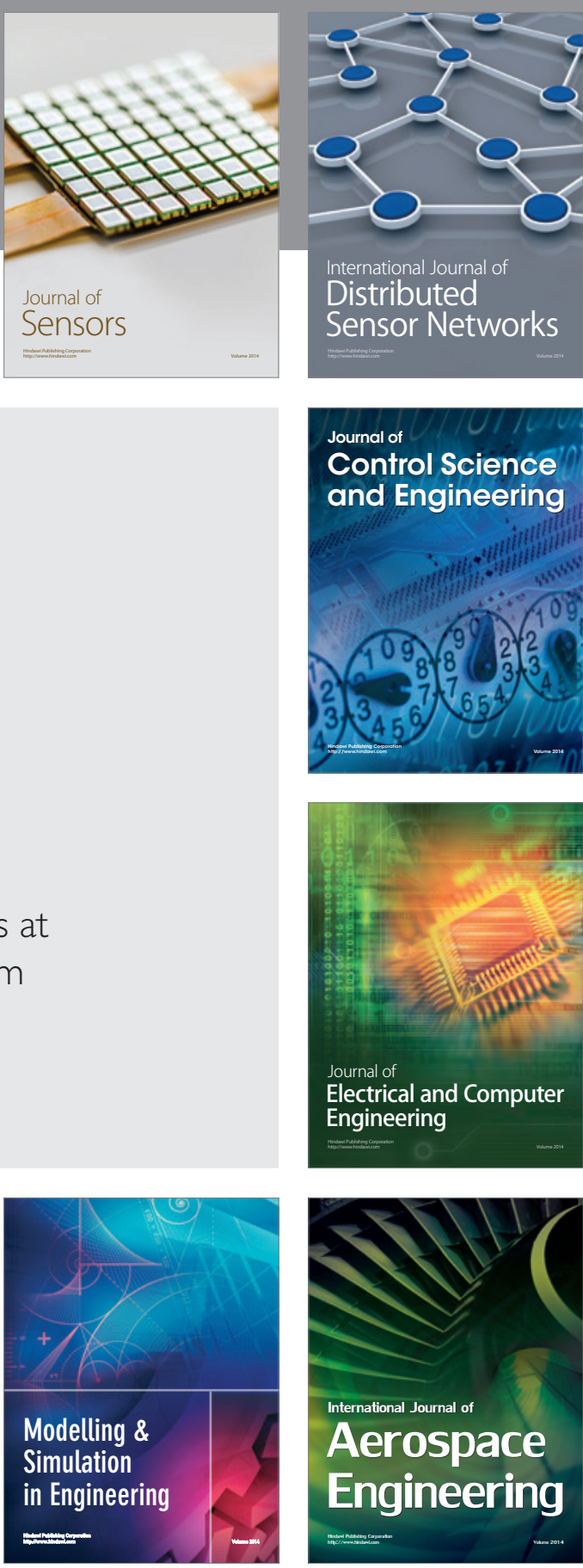

International Journal of

Distributed

Sensor Networks

Journal of

Control Science

and Engineering
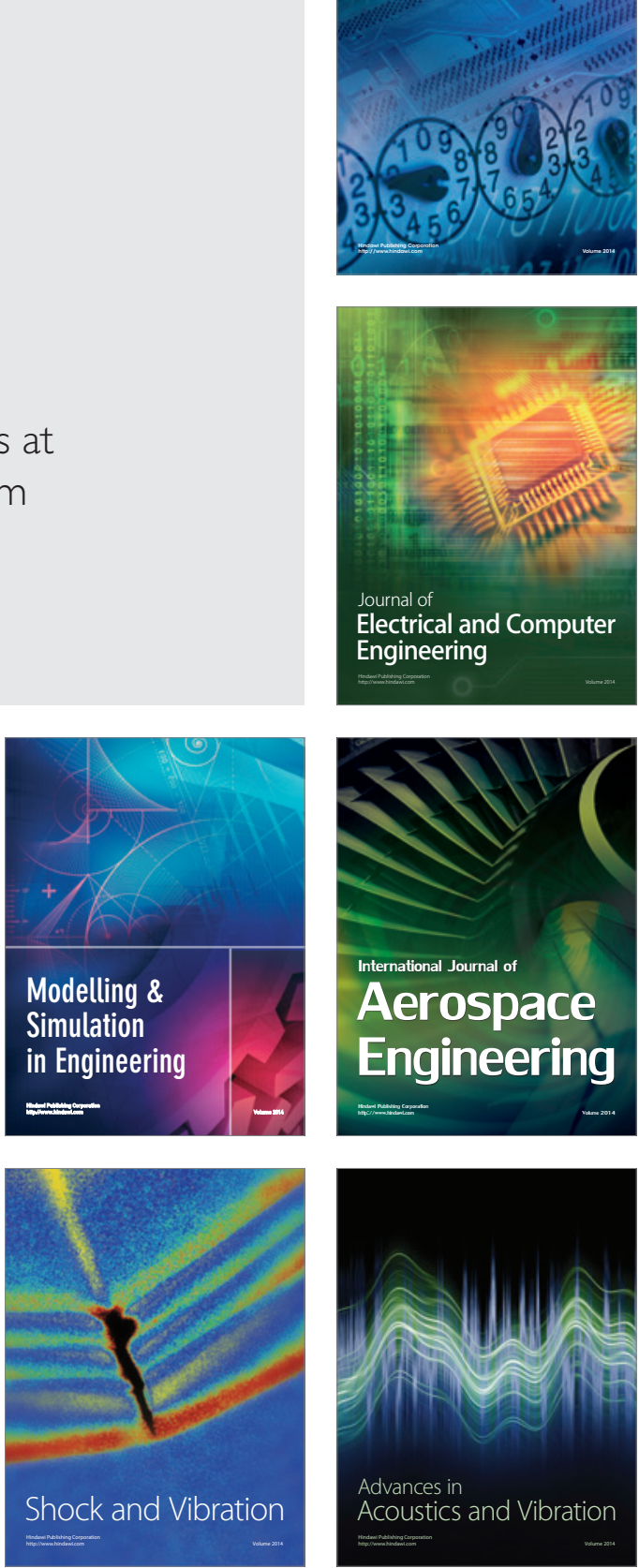